\title{
Guía para la valoración multiaxial del “esguince cervical" y la patología discal asociada
}

\section{Multiaxial guideline for evaluation of whiplash and associated disk disorders}

\author{
J. Aso Escario ${ }^{1}$ \\ JV. Martínez Quiñones ${ }^{1}$ \\ JL. De Miguel ${ }^{2}$ \\ A. Aso-Vizán ${ }^{3}$ \\ R. Arregui Calvo ${ }^{1}$
}

${ }^{1}$ Servicio de Neurocirugía, Hospital MAZ.

${ }^{2}$ Instituto de Investigación del Automóvil,

Centro Zaragoza.

${ }^{3}$ Servicio de Traumatología, Hospital Universitario Miguel Servet. Zaragoza

Correspondencia: José Aso Escario Servicio de Neurocirugía. Hospital MAZ

Avenida de la Academia General Militar 75, 50009 Zaragoza

E-mail: jaso@maz.es

Fecha de recepción:

13. MAR. 2014

Fecha de aceptación: 20. MAR. 2014

\section{Resumen}

Se aporta un enfoque de la valoración del esguince cervical basado en un modelo multidimensional. A modo de checklist se consideran aspectos relativos al accidente, las lesiones cervicales, el estado anterior, las lesiones extracervicales y aspectos psicológicos, incluyendo la simulación. Ya que el esguince cervical es no sólo, ni a veces principalmente, un cuadro médico, sino derivado de factores no médicos, se considera este modelo multiaxial como la mejor manera de abordar su valoración y tratamiento. Se insiste en la necesidad de valorar pericialmente las pruebas complementarias, en la importancia de algunas patologías asociadas y en la utilidad de un lenguaje común al hablar de la simulación o la exageración.

Palabras clave: Esguince cervical. Multidimensional. Simulación. Patologías asociadas al esguince cervical. Líneas guía. Valoración experta.

\begin{abstract}
A multiaxial approach to whiplash assessment is proposed. Following a checklist-like model, the accident, cervical and extra-cervical injuries, premorbid spine status, and psychological-malingering factors are, consecutively, considered. Provided that whiplash is not only a medical condition, but derived also from non-biological elements, the multiaxial model is presented as the best way to approach the forensic and therapeutic problems of whiplash. Remarks are made on the assessment of complementary tests under an expertise point of view, the importance of several whiplash-associated conditions, and the usefulness of using a unified terminology when facing malingering or simulation.
\end{abstract}

Key words: Whiplash. Multidimensional. Malingering. Whiplash associated disorders. Guidelines. Medical expertise.

\section{Introducción}

El enfoque actual del esguince cervical se basa en que el cuadro deriva no sólo de lesiones físicas, sino también de otros factores ${ }^{1}$. Una premisa, por tanto, es que las lesiones cervicales sufridas no justifican la evolución ni las secuelas de muchos de estos esguinces. En consecuencia, como primer aspecto en la investigación de este problema, falta un modelo conceptual que permita explicar globalmente los diferentes cuadros. Nuestra propuesta en este sentido es abordar el esguince cervical bajo un enfoque multiaxial ${ }^{2,3}$.

\section{Guía de valoración}

Para evaluar apropiadamente un esguince cervical consideraremos sucesivamente los siguientes ejes, que usaremos a modo de ckeck list en cada caso:

- Eje I: el accidente.

- Eje II: las lesiones físicas del raquis cervical.

- Eje III: el estado anterior.

- Eje IV: las lesiones asociadas fuera del raquis cervical.

- Eje V: los factores psicológicos, la simulaciónexageración. 
Hay un elemento adicional que no entra dentro de los mencionados ejes y que veremos aparte: la causalidad medicolegal.

Veamos, pues, cómo podemos aplicar en la práctica este esquema, en particular en los esguinces cervicales que superen los estándares habituales de estabilización (entre 1 y 3 meses como máximo).

\section{Eje I: ¿cómo fue el accidente?}

El médico forense debe buscar información suficiente sobre el accidente. Ello nos obliga a prestar atención y recopilar información sobre este evento (atestado, información del paciente, allegados, testigos, informes técnicos, etc.). Como ideas fundamentales al respecto proponemos las siguientes:

- El análisis de las solicitaciones (aceleraciones, esfuerzos y momentos) experimentadas por el raquis de los ocupantes de un vehículo, durante una colisión, pone de manifiesto que el riesgo de lesiones en el raquis está relacionado con la violencia de la colisión sufrida, por lo que un accidente de baja energía es poco probable que produzca lesiones sustanciales en ausencia de concausas.

- Sin embargo, no puede descartarse científicamente que accidentes de baja energía puedan causar lesiones (cervicales o extracervicales) o agravar estados anteriores, por lo que cuando esta situación se presente deberán buscarse las causas en el resto de los factores que se expondrán a continuación.

- Algunos elementos actúan amplificando o favoreciendo la aparición de lesiones: situación de la cabeza (si estaba rotada hay mayores repercusiones), reposacabezas mal ajustado.

- Un mecanismo a menudo olvidado son las elongaciones neurales y los traumatismos focales de nervios de las extremidades superiores. Pacientes con fuerzas actuando sobre las extremidades superiores pueden experimentar estiramientos de raíces o de troncos nerviosos. Por ejemplo, las manos sujetando el volante fuertemente pueden ser origen de neuropatías focales en los troncos nerviosos. También vaivenes notables cefálicos o de las extremidades superiores pueden causar mecanismos tipo "Burner" (cuadro descrito en futbolistas americanos que incluye desviación lateral de la cabeza y descenso del hombro, con dolor y disestesias en las extremidades superiores debido a elongación radicular extrarraquídea). Se trata de indagar sobre estas posibilidades tomando como referencia la información que podamos obtener del accidente.
- En conclusión, admitiendo que existe una relación entre la violencia de la colisión y el riesgo de lesiones por esguince cervical, cuando dicho esguince cervical no pueda ser explicado por el efecto de un agente traumático sobre un sujeto resultará necesario valorar otros elementos, destacando que los datos del accidente tienen gran importancia y deben ser recogidos, analizados por expertos en biomecánica y tenidos en cuenta dentro de un enfoque multiaxial.

\section{Eje II: las lesiones físicas del raquis cervical. ¿Cuáles son las lesiones cervicales objetivables?}

Se requiere una apropiada valoración de las lesiones, lo cual implica:

- Interpretación de la radiología, principalmente la resonancia magnética (RM), desde un punto de vista pericial y no sólo asistencial. Los informes asistenciales tienen un vicio de origen en tanto atienden a una finalidad asistencial. Por ello, muchas veces se omiten datos de interés pericial (signos de Modic, artrosis, desgarros anulares discales agudos, lesiones de partes blandas, etc.). Es preciso analizar "pericialmente" la radiología. Ello incluye:

- Examinar las placas (a ser posible los ficheros DICOM) y no contentarse sólo con el informe.

- Valorar las lesiones agudas en la RM. Alteración de señal en ligamentos, subluxaciones, desgarros o fisuras discales (signo HIZ), signos de estado anterior (signos de Modic, artrosis, redundancia, telescopaje, etc.).

- Realización de estudios dinámicos (radiología simple principalmente), valorar inestabilidades.

- Valorar las hernias/protrusiones discales según la clasificación de Coin ${ }^{4,5}$ (Tabla 1 y Figura 1). Las más frecuentes son las hernias de tipo 2 y tipo 3. El resto no deberían incluirse en el concepto de hernia traumática (el tipo 1 es excepcional y requiere muy elevada energía).

\section{Eje III: el estado anterior. ¿Existen concausas preexistentes?}

\section{Antecedentes}

Recabar datos en instituciones de salud. Muchas personas tienen antecedentes de cervicalgia. Algunos han tenido accidentes previos. Tienen valor los 
Tabla 1.

Clasificación de la hernia discal según Coin ${ }^{4,5}$.
Sujetos jóvenes

Traumatismos graves en flexión o extensión

Normalmente esguince grave o fractura-luxación asociados RM: afectación ligamentaria y partes blandas

Tipo 2. Protrusión discal

Expansiones discales difusas, con gran radio de curvatura

La degeneración y la debilidad del anillo fibroso crean los "discos de riesgo" que pueden llevar a lesiones de tipo 3

Tipo 3. Hernia aguda sobre disco degenerado

El material discal degenerado migra a través del anillo frágil o roto, produciendo una hernia "blanda"

Este tipo de hernia puede aparecer espontáneamente o ser favorecido por un traumatismo

Tipo 4. Hernia "crónica" y disco degenerado

Es la hernia "dura", asociada a calcificaciones ligamentarias y osteofitos

Este tipo de hernia es el precursor del tipo siguiente

Tipo 5. Uncodiscartrosis cervical

Proceso de degeneración crónica: estrechamiento del canal cervical y hernia de uno o varios discos con osteofitos asociados generalmente a una degeneración de las articulaciones interapofisarias posteriores

Figura 1.

Hernia discal aguda C5-C6

(flecha larga en a).

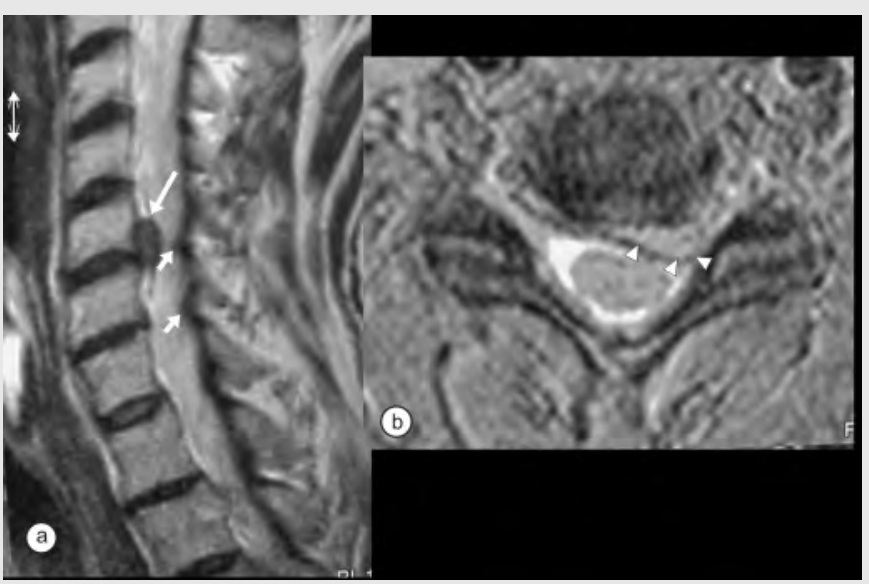

La redundancia ligamentaria, el pinzamiento y el aspecto oscuro en T2 del disco C6-C7 y superiores atestiguan un estado anterior degenerativo. Este caso corresponde al tipo 3 de Coin. En b se aprecia la hernia "blanda", más clara, deformando la médula (puntas de flecha).

Figura 2. Influencia del estado anterior.

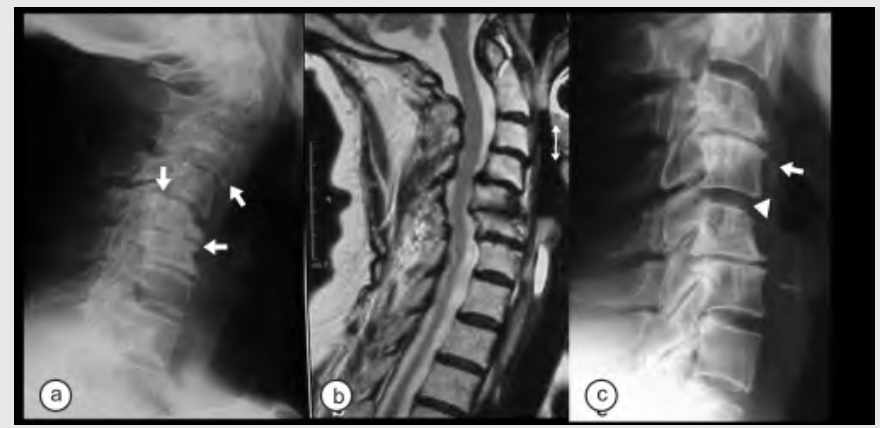

En a y b (mismo caso) se aprecia un bloque rígido C5-C6 (punta de flecha). En el accidente, este estado anterior fuerza a los segmentos adyacentes móviles a absorber toda la energía del impacto (flechas en a), condicionando luxaciones o subluxaciones ante energías a veces poco intensas. La imagen de b es la RM de este mismo caso. En $c$ vemos otro paciente, esta vez con dos bloques rígidos (flechas). En este lesionado, un traumatismo de baja energía dañó notablemente el disco interpuesto (punta de flecha), condicionando una mala evolución y un difícil tratamiento quirúrgico. 
datos clínicos informatizados, pues permiten conocer antecedentes que el paciente omite.

\section{Edad}

Las personas mayores tienden más a cuadros vestibulares o cordonales. Descartar en la RM cuadros de tipo Schneider (mielopatía poscontusiva por osteofitos posteriores).

\section{Artrosis}

Si hay artrosis suele haber segmentos rígidos. Ello implica una mala absorción biomecánica de fuerzas y la posibilidad de subluxaciones con baja energía (Figura 2). También hay estenosis de recesos laterales (con lo que hay propensión a la elongación radicular). Igualmente, en la espondiloartrosis hay una mayor propensión a alteraciones del simpático cervical (mareos, vértigos, etc.). Valorar uncoartrosis. Considerar la posibilidad de Doppler o angio-RM. En algún caso ha sido de utilidad la tomografía computarizada por emisión de fotón único (SPECT): el sujeto alegaba vértigos incoercibles y la normalidad de esta prueba descartó cualquier origen vascular cerebral.

\section{Eje IV: las lesiones fuera del raquis cervical. ¿Hay lesiones asociadas?}

Algunas lesiones muy frecuentes que pasan desapercibidas son:

- Síndrome de encrucijada cervicotorácica (TOS, thoracic oulet syndrome).

- Periartritis escapulohumeral.

- Síndromes neuromusculares. La contractura mantenida produce fibrosis muscular y pinzamiento de nervios locales (supraescapular, Arnold, etc.).

Todos estos cuadros deben responder al tratamiento. Ahora bien, necesitan ser identificados, tratados apropiadamente $\mathrm{y}$, sobre todo, no ser imputados a hernias de disco (bastantes pacientes terminan con artrodesis innecesarias).

Como métodos de diagnóstico están la clínica y exploración. Creemos muy importante el dúplex de troncos con maniobras de provocación ${ }^{6}$. La electromiografía (EMG) en busca de semiología troncular, o multirradicular posganglionar, si es preciso con potenciales evocados, resulta útil. Por ejemplo, una persona con una hernia discal cervical tendrá clínica y EMG de una, o como mucho dos, raíces del mismo lado. Si hay clínica o hallazgos EMG bilaterales y multirradiculares, esto no resulta imputable a una hernia moderada de un nivel, sino que hay que excluir

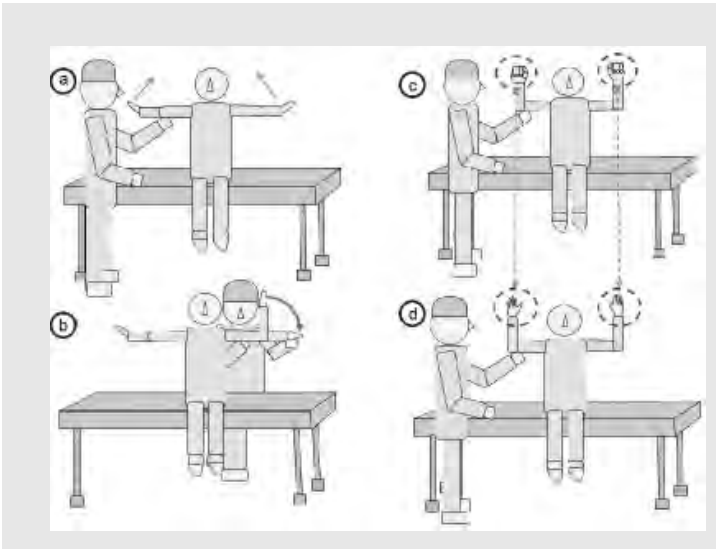

Figura 3.

Maniobras de estiramiento radicular de las extremidades superiores.
En a se representa la maniobra de Elvey, con los brazos extendidos y las manos con la palma hacia abajo, realizando una hiperextensión dorsal. En b vemos la llamada maniobra de Lasegue del miembro superior. El brazo, en ángulo recto, realiza una extensión con la palma hacia arriba. Los resultados de ambas maniobras persiguen valorar la existencia de dolor irradiado por el territorio neural. En c y d se representa la maniobra de Roos. En realidad, es una maniobra de desfiladero cervicotorácico. En posición de "manos arriba" se pide al paciente que extienda los dedos y después que cierre el puño, alternando ambas posiciones (abrir y cerrar la mano). La presencia de dolor o claudicación pone sobre la pista de un síndrome de encrucijada cervicotorácica. cuadros posganglionares (p. ej., TOS) o elongaciones radiculoplexulares.

\section{¿Cómo repercuten las lesiones cervicales y extracervicales en el sujeto?}

Un caso puede tener múltiples hallazgos radiológicos o EMG, pero diferente repercusión clínica; algo así como ocurre en el análisis de capacidad medicolegal. El criterio psiquiátrico puede demostrar una esquizofrenia, pero el llamado criterio psicológico (modo en que la enfermedad repercute en el sujeto) puede denotar una nula afectación de la capacidad. Aquí la exploración clínica resulta imprescindible.

\section{Exploración clínica}

- Maniobras de estiramiento radicular cervical: se conocen como upper limb tension test (ULTT) y fueron descritas por primera vez por Elvey ${ }^{7}$ (Figura 3). Nos dicen si hay repercusión radicular o no. Existen cuatro tipos que comprueban nervios y raíces, aunque hay que valorar sus resultados con cautela ${ }^{8}$.

- Reflejos: dan una idea de la afectación estructural de las raíces. Una indemnidad de reflejos va en contra de una lesión radicular de importancia. 
- Fuerza: Hay que explorarla segmentariamente. Una indemnidad de fuerza va en contra de una lesión radicular de importancia.

- Sensibilidad: valorar la sensibilidad exteroceptiva y propioceptiva (palestesia o sensibilidad vibratoria). Cuidado con sus interpretaciones, pues es muy subjetiva. Debe valorarse la distribución metamérica correcta.

- Maniobras vestibulares: hay tres maniobras importantes:

- Romberg: inespecífica (lesión vestibular o cordonal posterior).

- Unterberger o marcha en estrella: caminando hacia delante y atrás sobre los pasos, sin abrir los ojos en ningún momento. También inespecífica, pero muy evocadora de la realidad y entidad de un vértigo. Es recomendable efectuarla siempre.

- Marcha en tándem: también inespecífica (más evocadora de lesión cordonal posterior).

Un mareo con negatividad de estas maniobras es muy improbable que tenga entidad signi- ficativa. Estas pruebas demuestran también conductas extrañas (estilo de respuesta no fidedigno: Romberg histérico).

- Artrometría cervical: se precisan elementos objetivos. En la práctica aconsejamos fotografías (tras pedir autorización al paciente) y medir luego tranquilamente sobre ellas los arcos de movilidad. Si se dispone de sistemas biomecánicos, mejor.

\section{Eje V: los factores psicológicos. La simulación-exageración. ¿Hay estilo de respuesta distorsionado?}

Estructurar el examen identificando inconsistencias (relato de síntomas sin entidad o inconsistentes), incongruencias (falta de concordancia entre dos maniobras de la exploración clínica), discrepancias (entre dos elementos diferentes de la historia clínica) y contradicciones (en la misma visita o entre dos visitas distintas) ${ }^{9}$.

Intentar clasificar el estilo en uno de los siguientes tres grupos:

Tabla 2.

\section{Relación causal}

\section{।}

II

III

IV

V

\section{Relación imposible de determinar}

Relación negativa: no existe relación valorable entre la causa y el efecto

Relación posible (entra dentro de la lógica médica, pero la relación no es documentable)

- Illa: entra dentro de la lógica médica, pero fuerza de relación incongruente (quiebra de numerosos criterios de concausalidad)

- Illb: entra dentro de la lógica médica, si bien la fuerza de relación es débil

Relación probable: hay alguna o algunas pruebas objetivas que en cada caso concreto pueden documentar la probabilidad de una relación causa-efecto

- IVa: fuerza de relación media

- IVb: fuerza de relación intensa

Relación inequívoca: hay pruebas médicas que en cada caso concreto determinan inequívocamente la relación

\section{Relación concausal}

Tipo I

Un efecto totalmente imputable a la causa

Tipo II

Un efecto nuevo por una causa y unas concausas

Tipo III

Una agravación de un estado anterior

Tipo IV
Una agravación del efecto por el estado anterior 
En a, b y d se muestran dispositivos intersomáticos, probablemente los más usados en la actualidad. Nótese, además, que pueden colocarse a un nivel, a dos (a) o combinarse con placas (c). En e se muestra el aspecto de una placa anterior. Lógicamente, el baremo debe tener en cuenta el tipo, la extensión, la multiplicidad y las características del material implantado, ya que, como vemos, existen grandes diferencias.

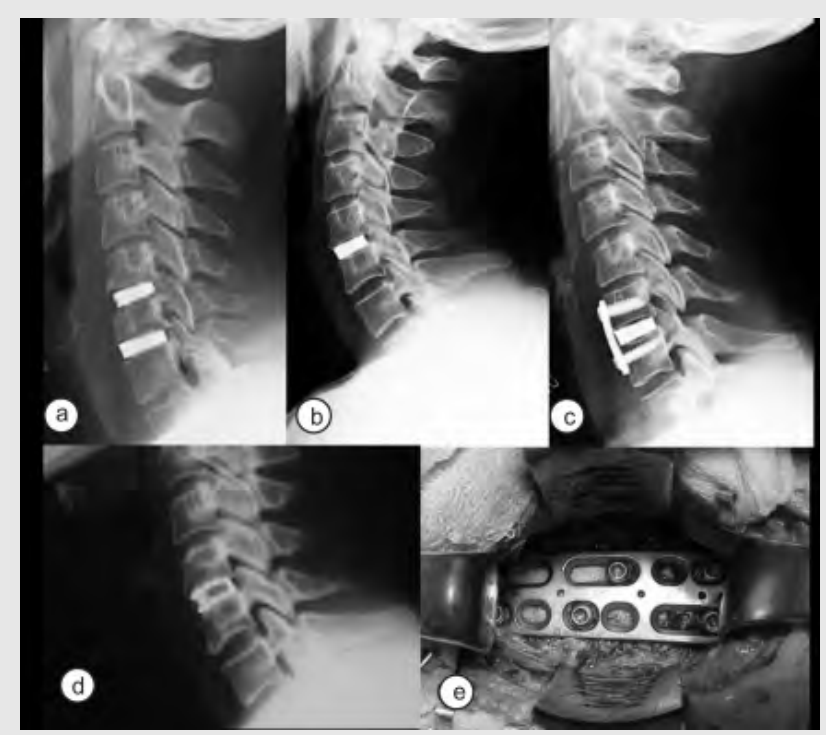

Figura 4.

Distintos tipos de material de artrodesis cervical.
- ERAD: estilo de respuesta amplificado o distorsionado. No hay claros incentivos, pero el sujeto muestra inconsistencias, incongruencias, discrepancias o contradicciones.

- ERNOF: estilo de respuesta no fidedigno. Sospecha de expectativa de ganancia y falta de fiabilidad, por el número o las características de los signos mencionados.

- SIVE: simulación verdadera. En ésta se cumplen los criterios DSM de simulación: intención deliberada de falsear o amplificar siguiendo un incentivo externo.

Hablando el mismo lenguaje y acumulando semiología de estos tipos, resulta fácil orientarse en el terreno de la simulación y plasmar los resultados en los informes de una manera homologada y coherente.

\section{Análisis causal}

Muchas veces se plantea la intervención y el peso reales del accidente en el resultado final. Esto es un análisis de causalidad medicolegal. Hay que usar los criterios de causalidad, entre los cuales los dos más aceptados son los de Simonin y los de Bradford Hill.

Para el análisis causal, proponemos nuestro modelo publicado para las hernias de disco ${ }^{10}$ (Tabla 2).

El modelo multiaxial puede servir para explicar el significado y el valor de algunos criterios (p. ej., el accidente en la proporcionalidad, las lesiones asociadas en el de exclusión y como concausas, el estado anterior al de exclusión, etc.).

\section{Cómo valorar las secuelas}

Nos referiremos al RDL 8/2004, en el que se contemplan las siguientes secuelas:

- Artrosis postraumática sin antecedentes (1-8): secuela imposible de valorar en un esguince. Podría darse en fracturas y con los años. Recomendamos no valorarla en el esguince cervical.

- Agravación de artrosis previa al traumatismo (15): secuela de formulación ambigua. Es preciso conocer el estado anterior del sujeto y esperar meses o años para ver agravamientos. Recomendamos no valorarla en el esguince cervical.

- Osteítis vertebral postraumática sin afectación medular (30-40): este cuadro es posfractura y no debe producirse en el esguince cervical. Recomendamos no valorarla en el esguince cervical.

- Material de osteosíntesis en la columna vertebral (5-15): el método de referencia de tratamiento de una discopatía-hernia tras un esguince cervical es la discectomía y fusión vertebral, bien con injerto-placa, bien con dispositivos intersomáticos (mucho más utilizados actualmente) (Figura 4). Algunos dispositivos permiten el 
movimiento (prótesis discales o artroplastia). Es recomendable baremar según el número de niveles con implante y la magnitud de éste (extensión, complejidad). No importa mucho el tipo de dispositivo, pues aunque se haga artroplastia, a largo plazo, suele tender a fusionarse.

- Considerar valorar junto con el implante dos complicaciones de dispositivos intersomáticos: settlement (hundimiento ligero del implante en el platillo) y subsidience (hundimiento significativo y progresivo del implante, con mala posición definitiva). Si se emplea placa atornillada, el valor en el rango podría ajustarse al alza, ya que es un material más complejo, extenso y sujeto a complicaciones. Importante aquí que el RDL valora el material, no la intervención de disco, por lo que habría que baremar aparte el epígrafe 6 , relativo a la hernia discal operada o sin operar.

- Fractura-acuñamiento anterior/aplastamiento: no tienen cabida en el esguince cervical.

- Cuadro clínico derivado de hernia/s o protrusión/ es discal/es operada/s o sin operar: se considera globalmente todo el segmento afectado de la columna (cervical, torácica o lumbar) (1-15). Se habla aquí del cuadro clínico. Un paciente puede tener una hernia en la RM y no tener clínica. También puede tener una artrodesis, pero no un cuadro clínico derivado de una hernia operada. La clínica derivada de una hernia discal incluye dolor local espontáneo o con los movimientos, y afectación radicular segmentaria (irritativa 0 deficitaria).

Para valorar el cuadro clínico aconsejamos tener en cuenta las maniobras que denotan afectación radicular (MER, ROT, fuerza, sensibilidad). La EMG ayuda. En teoría esta secuela incluiría el algia postraumática, por lo que podemos valorar como apartado 6 o como apartado 8, pero no ambos a la vez, siempre que el paciente no presente otros signos de afectación radicular (el algia es sólo uno de ellos).

Recomendamos utilizar este apartado para hernias documentadas que explican coherentemente la afectación segmentaria y radicular, o bien para pacientes operados con dolor posterior y exploración coherente. Si la clínica, la radiología y la exploración no permiten una imputación clara a una afectación discal, el perito debe considerar su valoración como algia postraumática (punto 8). Hay que hacer notar que el baremo del punto 6 queda un poco justo en los déficits motores, ya que contempla el déficit motor con rangos mucho más altos en otros epígrafes (paresia de algún grupo muscular: 5-25). En consecuencia, nuestra recomendación sería baremar aparte los déficits motores imputables a lesión radicular discal.

- Alteraciones de la estática vertebral posfractura (valor según arco de curvatura y grados) (1-20): no tiene aplicación en el esguince.

- Algias postraumáticas:

- Sin compromiso radicular (1-5).

- Con compromiso radicular (5-10).

Para valorar el compromiso radicular aconsejamos las MER, la exploración de reflejos, fuerza y sensibilidad, y la EMG.

- Columna cervical: limitación de la movilidad de la columna cervical (5-15). Recomendamos baremarla aparte del material de osteosíntesis, ya que el epígrafe 4 antes mencionado no barema la fusión vertebral sino el material. En nuestra opinión, dada la variabilidad en causas y en repercusión de éstas sobre la movilidad cervical, recomendamos valorar esta última aparte del resto de los epígrafes.

- Síndrome postraumático cervical (cervicalgia, mareos, vértigos, cefaleas) (1-8): este epígrafe debería reservarse a cuadros de tipo vestibular o simpático cervical (mareos, inestabilidad, etc.). Sugerimos comprobarlo con pruebas como Romberg, tándem o Unterberger, o bien mediante pruebas especiales como Doppler, angio-RM/ TC o SPECT). No aconsejamos baremar en este epígrafe cuando el paciente presenta poco más que cefalea.

\section{¿Qué permite el modelo multiaxial?}

- Explorar todas las causas posibles de prolongación de la estabilización de un esguince más allá de 1-3 meses, que se considera la duración normal o promedio.

- Tratar las causas identificadas que, de otro modo, pasarían desapercibidas.

- No cometer errores y artrodesar casos que no lo requieren (tienen lesiones posganglionares 0 extracervicales y no intracervicales).

- Limitar el distrés iatrogénico que resulta de la imputación de los síntomas a una simulación o intento fraudulento en los casos en que estos últimos no existen.

- Hallar estilos de respuesta distorsionados o no fidedignos, y exponer su naturaleza y causas en un informe medicolegal con un lenguaje unitario. 
- Baremar apropiadamente un caso concediendo a cada eje el peso que realmente tiene en la evolución del caso.

- Ofrecer un modo común de valoración de estos cuadros al objeto de unificar criterios y construir una referencia para el estudio de poblaciones de una manera homologada.

\section{Agradecimientos}

A Luis Berdusán (Hospital MAZ) por su ayuda para la realización de las ilustraciones.

Los autores declaran que el presente trabajo no ha sido subvencionado total ni parcialmente por ninguna entidad pública o privada, y que no existe ningún tipo de conflicto de intereses.

\section{Bibliografía}

1. Giannoudis PV, Mehta SS, Tsiridis E. Incidence and outcome of whiplash injury after multiple trauma. Spine. 2007;32(7):776-81.

2. Proyecto Prelace. Investigación para el desarrollo de tecnologías más eficaces en la prevención del latigazo cervical. Madrid: Ministerio de Industria, Turismo y Comercio; 2008. IAP-560410-200861.

3. Aso J, Martínez Quiñones JV. Patología traumática de columna vertebral. En: Delgado S, editor. Tratado de medicina legal y ciencias forenses. Tomo II. Barcelona: Editorial Bosch; 2012.

4. Coin CG. Cervical disc degeneration and herniation. Diagnosis by computerized Tomography. South Medical J. 1984;77:979.

5. Coin CG, Coin JT, Garrett JK. Computed tomography of canine disc herniation: a potential diagnostic model for the evaluation of disc disease and discolysis in humans. En: Donovan MJ, editor. Computed tomography of the spine. Baltimore: Williams \& Wilkins; 1984. pp. 471-6.
6. Aso J, Pérez Monreal J, Martínez Quiñones JV, Arredondo JM, Arregui R. Síndrome de desfiladero cervicotorácico postraumático y traumatismos cervicales menores. Importancia médico-legal. Revista Española de Medicina Legal. 2009;35(2):51-8.

7. Elvey RL. Brachial plexus tension tests and the pathoanatomical origin of arm pain. En: Glasgow $\mathrm{EF}$, Twomey L, editores. Aspects of manipulative therapy. Melbourne: Lincoln Institute of Health Sciences; 1979. pp. 105-10.

8. Kleinrensink GJ, Stoeckart R, Mulder PG, Hoek G, Broek T, Vleeming A, et al. Upper limb tension tests as tools in the diagnosis of nerve and plexus lesions. Anatomical and biomechanical aspects. Clin Biomech (Bristol, Avon). 2000;15(1):9-14.

9. Aso J, Martínez Quiñones JV, Arregui R. Simulación en patología espinal. Madrid: Grupo 2 Eds. 1994.

10. Aso J, Martínez-Quiñones JV, Consolini F, Domínguez M, Arregui R. Hernia discal traumática. Implicaciones médico-legales. Cuad Med Forense. 2010;16(1-2):19-30. 